\title{
A consumer charter for genomic services
}

\author{
Fred Ledley
}

Within this decade, the identification of discrete genetic factors involved in healthy development and disease will become routine, providing the foundation for truly personalized medicine in which individuals are empowered not only with self-knowledge of their genetic risk, but also with the ability to take informed actions to prevent disease and preserve health. Currently, the healthcare system lacks the capacity to deliver quality genetic services of this type at the scale that will be required. I believe several principles are required for the move to a consumer-focused model for genetic services.

\section{The charter}

Provide information. Consumers need reliable sources of information that describe specific genetic tests as well as the actions that they could take in response to a test result to protect health or prevent disease. This information must be at a comprehension level appropriate for the general public and should emphasize medical issues and actions rather than basic genetics. Importantly, such information should help consumers distinguish between basic genetic discoveries, tests in research or development, and validated tests with established clinical indications. The Internet provides an ideal medium for communicating information directly to large numbers of consumers. Such information would ideally be coordinated with conventional informational materials that could be distributed by healthcare providers to their patients and educational materials directed at the providers themselves.

Informed consent. The informed consent process provides a powerful opportunity for a structured dialogue between consumers and providers concerning genetic tests, a vehicle through which consumers can explicitly direct the process of genetic testing, and a structure that can be built upon to ensure individual autonomy and the integrity of genetic services.

The utility and quality of the current consent process for genetic tests can be upgraded by developing clinically validated instruments that give consumers information about a specific genetic test and give providers stepwise instructions on how to present this informa-

Fred D. Ledley is Founder and Chairman of Mygenome, Needham, MA

(fledley@mygenome.com). tion to consumers, assess the consumer's understanding, and obtain valid consent. An informed consent instrument should describe, in a nondirective manner, the availability and applicability of a genetic test, its potential benefits and risks, the process of performing the test, policies governing the disposition and use of clinical data and clinical samples, provisions for protecting privacy, and mechanisms for follow-up.

The formal consent document itself should enable consumers not only to authorize a genetic test, but also to authorize specific practices regarding the use of clinical information and samples. Instruments for informed consent may employ advanced information and interactive technologies or printed workbooks for use by healthcare providers with their patients. Instruments can also be developed that enable consumers to provide informed consent without any direct involvement of a healthcare provider by reviewing information, completing a self-assessment, and indicating their consent.

Direct access to testing. Every individual has a fundamental right to obtain genetic information about himself or herself in a confidential manner by having genetic tests performed privately and receiving the test results directly from a testing laboratory. The consumer-focused model empowers consumers with direct and private access to genetic tests and services. To ensure quality, direct consumer services should be coupled to a valid informed consent process and sources of counseling, follow-up, and referrals to specialty healthcare providers. Giving consumers the option to claim reimbursement or to pay out of pocket for genetic tests can further enhance consumer choice and privacy.

Personal control. Given the fragmentation of medical care and medical records among many healthcare providers, it is essential that consumers have control over this information and the ability to bring it to the attention of their healthcare providers. Moreover, empowering consumers with responsibility for their own genetic information would resolve difficult issues regarding privacy by enabling each individual to determine what level of privacy is appropriate for him or her. Many individuals may choose to have personal genetic information incorporated in their medical records or used for research, thus accepting any attendant risks to privacy. Others may choose to keep this information confidential or release it selectively when it impacts their healthcare.
Reassess regulations. Regulations on genetic testing must be reassessed to ensure quality without restricting access to the potential health benefits of genomics. Regulations that require a physician prescription for genetic tests should be replaced with a requirement for certified informed consent. Regulations that prohibit laboratories from reporting results directly to consumers should be eliminated. Such restrictions do not allow consumers to decide who should have access to their personal genetic information and seriously compromise protections for personal privacy. Also, regulations should not inhibit the free communication and marketing of information concerning selected genetic tests directly to consumers other than to ensure the integrity of such information.

Generate data to guide consumer choice. To make informed decisions regarding genetic tests, consumers need information on the positive predictive value of genetic tests, the personal costs, risks, and benefits of genetic testing, and the actions that may be taken to prevent disease. Many clinical studies and professional recommendations concerning testing, however, focus on issues of disease prevalence, genetic epidemiology, healthcare utilization, and pharmacoeconomics, which are relevant to payers and providers. New research is required to generate data that will allow consumers to prioritize genetic tests in the context of multifaceted, environmental, medical, and lifestyle choices. Research is also required to understand how consumers make choices regarding genetic tests, and how healthcare providers can help to ensure that these choices are informed and effective.

\section{Conclusions}

Inherent barriers in the current healthcare system could inhibit the application of genomics to personal health. A radical reassessment of healthcare delivery models is necessary to ensure that the health benefits of genomics are realized by the greatest number of individuals in the shortest possible time. A model that empowers consumers with confidential knowledge of their own genome and the ability to make informed decisions concerning their healthcare represents the ultimate expression of a truly personalized medicine. It is also an effective strategy for circumventing the inadequate infrastructure for genomic services, improving the quality of care, and strengthening protections for individual privacy and autonomy. 